\title{
Ab initiostudies of the phase transitions inK2Se04
}

\section{Citation}

Lu, H. M., and J. R. Hardy. 1990. "Ab Initiostudies of the Phase Transitions inK2Se04." Physical Review B 42 (13) (November 1): 8339-8350. doi:10.1103/physrevb.42.8339.

\section{Published Version}

doi:10.1103/PhysRevB.42.8339

\section{Permanent link}

http://nrs.harvard.edu/urn-3:HUL.InstRepos:32417867

\section{Terms of Use}

This article was downloaded from Harvard University's DASH repository, and is made available under the terms and conditions applicable to Other Posted Material, as set forth at http:// nrs.harvard.edu/urn-3:HUL.InstRepos:dash.current.terms-of-use\#LAA

\section{Share Your Story}

The Harvard community has made this article openly available.

Please share how this access benefits you. Submit a story.

Accessibility 


\title{
$A b$ initio studies of the phase transitions in $\mathrm{K}_{2} \mathrm{SeO}_{4}$
}

\author{
H. M. Lu and J. R. Hardy \\ Department of Physics and Center for Electro-Optics, University of Nebraska-Lincoln, Lincoln, Nebraska 68588-0111
}

(Received 18 June 1990)

\begin{abstract}
An ab initio model is developed for the potentials in ionic molecular solids in which the electron covalency within the molecular ions substantially affects the interionic interactions. By treating the intermolecular and intramolecular interactions on the basis of the true electron charge densities of the molecular ions, this new model leads to an accurate parameter-free description of the potentialenergy surfaces for such crystals. We performed first-principles static structural relaxation, supercell molecular-dynamics simulation, and lattice-dynamics studies for the room-temperature paraelectric phase and the lower-temperature ferroelectric superstructure of $\mathrm{K}_{2} \mathrm{SeO}_{4}$ and predicted with good accuracy the transition from the former to the latter. Given the excellent agreement between theory and experiment, we then explored the delicately balanced potential-energy surfaces for $\mathrm{K}_{2} \mathrm{SeO}_{4}$ and found that they contain a double-well type of structure which is the essential origin of the incommensurate and the subsequent commensurate transitions in $\mathrm{K}_{2} \mathrm{SeO}_{4}$.
\end{abstract}

\section{INTRODUCTION}

As one of the earliest insulators to exhibit an incommensurate phase, potassium selenate $\left(\mathrm{K}_{2} \mathrm{SeO}_{4}\right)$ has attracted extensive experimental and theoretical efforts. ${ }^{1}$ In the room-temperature (paraelectric) phase the crystal is orthorhombic with space-group Pnam $\left(D_{2 h}^{16}\right) .^{2}$ At $129.5 \mathrm{~K}$ it undergoes a displacive type of structural phase transition into an incommensurate phase, as is evidenced by the presence of satellite peaks in $x-$ ray $^{3}$ and neutronscattering ${ }^{4}$ studies. The neutron experiments show that this structural instability is driven by a soft phonon with a wave vector $q=\frac{1}{3}\left[(1-\delta) \mathbf{a}^{*}\right]$, where $\mathbf{a}^{*}$ is the first reciprocal-lattice vector along the [100] direction. As the crystal is cooled further, $\delta$ decreases and vanishes abruptly at $93 \mathrm{~K}$, when the system locks into a commensurate superstructure whose a axis is triple the size of that of the room-temperature phase. The structure of this new phase is also orthorhombic with space group Pna $2_{1}\left(C_{2 v}^{9}\right)$ and the crystal acquires a spontaneous polarization along the $\mathrm{c}$ axis.

The first theoretical work on a microscopic basis for $\mathrm{K}_{2} \mathrm{SeO}_{4}$ was by Haque and $\mathrm{Hardy}^{5}$ With the shortrange forces in a rigid-ion model determined by using the equilibrium constants and the observed Brillouin-zonecenter frequencies, they performed lattice-dynamics studies on the basis of a quasiharmonic approximation and found that the lowest-lying $\Sigma_{2}$ branch indeed has a minimum near $q=a^{*} / 3$, in agreement with the neutronscattering results. ${ }^{4}$ An important finding of this work is the fact that the origin of the instability responsible for the incommensurate and the ferroelectric phase transitions is the near cancellation between large Coulomb and short-range forces. This suggests that a reliable understanding of the transition mechanism has to be based on an accurate description of both components of the interionic interactions, and thus of their differences.

In this paper we describe first-principle simulations of
$\mathrm{K}_{2} \mathrm{SeO}_{4}$ using $a b$ initio interionic potentials. Such simulations have proved very successful in explaining melting, ${ }^{6}$ superionicity, ${ }^{7}$ zone-boundary instabilities in halide perovskites, ${ }^{8,9}$ as well as incommensurate phase transitions. ${ }^{10-12}$ By using the Gordon-Kim electron-gas mod$\mathrm{el}^{13}$ to compute the short-range potentials directly from $a b$ initio electron charge-density distributions of the individual ions, this approach provides, within the rigid-ion and pairwise interaction approximation, realistic potential-energy surfaces for crystals and therefore allows clear and reliable discrimination between delicately balanced structural instabilities.

The most basic assumption underlying the original Gordon-Kim model is that, for closed-shell ions, the total electron charge-density distribution of an ion pair is a superposition of the charge densities of the individual ions. While such an assumption seems valid for strictly ionic crystals, it becomes inapplicable to crystals that contain molecular ions, i.e., ionic molecular crystals, to which class $\mathrm{K}_{2} \mathrm{SeO}_{4}$ belongs. More specifically, this assumption could be invalid when the ion pair in question consists of atoms that are parts of the same molecular ion, e.g., an $\mathrm{Se}-\mathrm{O}$ pair within the selenate ion $\mathrm{SeO}_{4}{ }^{2-}$. This is so because there could be marked electron covalency within the molecular ions, as is certainly the case for the selenate. On one hand, this covalency renders the total electron charge distribution inexpressible by a simple superposition of the electron charge distributions of the constituent atoms and therefore prohibits the use of Gordon-Kim potentials for the intramolecular interactions. On the other hand, the covalency alters the electronic populations on the individual atoms substantially from the closed-shell configurations so that interactions external to the molecular ions are also affected. Moreover, the electron covalency introduces many-body and/or noncentral interactions, which fall outside of the scope of the usual pairwise-central-force scheme.

In fact, such an inadequacy of the conventional ap- 
proach has been found during a study of phase transitions in $\mathrm{Rb}_{2} \mathrm{ZnCl}_{4} \cdot{ }^{10-12}$ Specifically, the short-range potential for the $\mathrm{Zn}^{2+}-\mathrm{Cl}^{-}$ion pair computed from the electron charge densities of the free $\mathrm{Zn}^{2+}$ and $\mathrm{Cl}^{-}$ions was manifestly too hard, and an overall reduction of this potential had to be adopted to give good agreement with the experimental $\mathrm{Zn}-\mathrm{Cl}$ bond length. Also some distortions of the $\mathrm{ZnCl}_{4}{ }^{2-}$ ion occurred, probably resulting from the combination of lack of intramolecular bond-angle stiffness and full ionicity. Moreover, the theoretical structures fail to give the correct orientations of the $\mathrm{ZnCl}_{4}{ }^{2-}$, which clearly indicates defects in the descriptions of the intermolecular interactions. This inadequacy of the method would become even more obvious if it were used to treat $\mathrm{K}_{2} \mathrm{SeO}_{4}$, since the required closed-shell electron charge-density distribution would give selenium the unrealistically large ionicity of $6+$.

In this work we present a major extension of the Gordon-Kim approach to treat ionic molecular crystals, as exemplified by $\mathrm{K}_{2} \mathrm{SeO}_{4}$. The essence of this is to treat the molecular ions as single entities and give separate considerations to the intramolecular and intermolecular interactions. The starting point of our new approach is to perform $a b$ initio quantum-chemistry calculations for the whole molecular ions, i.e., $\mathrm{SeO}_{4}{ }^{2-}$. From these calculations one obtains an energy expression for the molecular ion to evaluate the intramolecular interactions, and one also obtains the electron charge distribution for the calculation of the intermolecular interactions. As will be shown, this approach produces very accurate potentialenergy surfaces for $\mathrm{K}_{2} \mathrm{SeO}_{4}$.

On the basis of the resultant ab initio potential-energy surface we first performed symmetry restricted static relaxations for the room-temperature Pnam and the lowertemperature $P n a 2_{1}$ structures of $\mathrm{K}_{2} \mathrm{SeO}_{4}$. Apart from an overall shorting of the lattice constants, which is a rather general feature for large unit-cell simulations using Gordon-Kim potentials, the theoretical structures agreed very well with the experimental data, indicating that our potentials are very satisfactory.

The method of molecular dynamics was then used to simulate directly the dynamical states of $\mathrm{K}_{2} \mathrm{SeO}_{4}$ at different temperatures. By quenching a supercell of 336 ions, we studied the transition of $\mathrm{K}_{2} \mathrm{SeO}_{4}$ from its roomtemperature Pnam structure to its lower-temperature $P n a 2_{1}$ structure. However, since this simulation uses periodic boundary conditions, it cannot directly simulate the incommensurate structure of $\mathrm{K}_{2} \mathrm{SeO}_{4}$. Nonetheless, since both the incommensurate and the subsequent ferroelectric lock-in transitions originate from the same basic structural instability, they appear as a combined transition in the simulation. By computing a conveniently defined order parameter for the simulated structures at different temperatures, we can infer temperatures for the onset and the lock-in of this transition. These temperatures match closely the experimental values to within the statistical uncertainties of the supercell moleculardynamics simulations associated with finite sample size.

To investigate the structural instability from a different point of view, we then calculated the dispersion curves for the Pnam structure of $\mathrm{K}_{2} \mathrm{SeO}_{4}$ along the $\mathbf{a}^{*}$ direction using our $a b$ initio potentials. It was found that the most unstable point indeed occurs near $\frac{1}{3} \mathbf{a}^{*}$, as is the case for experiment.

Based on this excellent agreement between static and dynamic simulations and the experimental data, we shall then discuss the origin of the structural instability responsible for the phase transitions. It will be shown that a double-well type of structure in the potential-energy surface for the rotation of the $\mathrm{SeO}_{4}{ }^{2-}$ ions is the driving mechanism of the transitions. A new and stable structure is found, which has a singlet rather than the triplet structure found experimentally. Although this new structure has a potential energy slightly lower than the triplet $P n a 2_{1}$ structure, it cannot be accessed by the supercell molecular-dynamics simulations. An explanation for this is given in terms of the difference between the entropies of the singlet and the triplet structures.

This paper is organized as follows: in Sec. II we present the procedure for obtaining the intramolecular and intermolecular potentials from quantum-chemistry calculations. Section III gives the results of the static relaxations for both the room-temperature Pnam and the lower-temperature $P$ na $2_{1}$ structures. In Sec. IV we describe molecular-dynamics calculations to simulate the phase transitions in $\mathrm{K}_{2} \mathrm{SeO}_{4}$. Section $\mathrm{V}$ considers qualitatively the lattice instability from the point of view of lattice dynamics and discusses the driving mechanism of the transition. Section VI concludes the paper. It should also be noted that a Brief Report of the present work has been published. ${ }^{14}$

\section{CALCULATIONS OF THE AB INITIO INTERMOLECULAR AND INTRAMOLECULAR POTENTIALS}

As pointed out in the Introduction, our starting point is to perform $a b$ initio quantum-chemistry calculations for the $\mathrm{SeO}_{4}{ }^{2-}$ molecular ion. Our calculations were made for an isolated $\mathrm{SeO}_{4}{ }^{2-}$ ion and thus did not include a background crystal field. This is justified by the finding that, while the oxygen sites in $\mathrm{K}_{2} \mathrm{SeO}_{4}$ are the only sites with nonnegligible crystal electric fields, over $90 \%$ of the contribution to these fields is from the other ions in the same $\mathrm{SeO}_{4}{ }^{2-}$ group, rather than from the rest of the lattice. This is found to be true for both the roomtemperatures Pnam and the lower-temperature Pna $2_{1}$ structures.

We first performed a quantum chemistry structural optimization for the $\mathrm{SeO}_{4}^{2-}$ ion, which searches for the atomic configuration that gives the lowest self-consistent Hartree-Fock energy. The optimized $\mathrm{SeO}_{4}{ }^{2-}$ ion forms a perfect tetrahedron with the $\mathrm{Se}-\mathrm{O}$ bond length being 3.09 a.u., very close to the experimental average value of 3.07 a.u. in the Pnam phase. At the optimized configuration, the force constants, i.e., the second derivatives of the total energy of the $\mathrm{SeO}_{4}{ }^{2-}$ ion with respect to distortions from this optimized structure, are then calculated. With these second derivatives, one can construct a harmonic expansion of the energy of the free $\mathrm{SeO}_{4}{ }^{2-}$ ion in terms of its bond lengths, bond angles, and dihedral angles. This expansion provides a convenient description 
of the intramolecular interactions, i.e., the $\mathrm{Se}-\mathrm{O}$ and $\mathrm{O}-\mathrm{O}$ interactions within the same $\mathrm{SeO}_{4}{ }^{2-}$ ion. Such a description is adequate as far as studies of the phase transitions are concerned, since throughout, in the Pnam, incommensurate, and $P_{n a} 2_{1}$ phases, the $\mathrm{SeO}_{4}{ }^{2-}$ ions stay as near perfect tetrahedra, in agreement with experiment.

These quantum-chemistry calculations were performed using the GAUSSIAN86 program. ${ }^{15}$ For the oxygen atoms the standard 6-31G basis set was used, and for Se atom, the STO-3G basis was employed. For both atoms single first polarization functions were added to the basis sets.

We now turn to intermolecular interactions, i.e., those between the ion pairs, K-Se, K-O, and Se-Se and the pairs $\mathrm{Se}-\mathrm{O}$ and $\mathrm{O}-\mathrm{O}$, where the ions in a pair belong the different molecular groups. For these ion pairs there is no electron covalency involved, therefore the GordonKim electron-gas model should still be appropriate for the calculation of these potentials. To perform these calculations we need the electron charge distributions of the individual $\mathrm{K}, \mathrm{Se}$, and $\mathrm{O}$ ions. For $\mathrm{K}$ we simply use the closed-shell charge distribution for free $\mathrm{K}$ ion, ${ }^{16}$ while for Se and $O$ we extract their charge densities from the charge density of the whole $\mathrm{SeO}_{4}{ }^{2-}$ ion obtained in the self-consistent Hartree-Fock calculation for the optimized structure of $\mathrm{SeO}_{4}{ }^{2-}$. Briefly, this extraction is made in the spirit of Mulliken population analysis. ${ }^{17}$ The total electron charge density for the whole $\mathrm{SeO}_{4}{ }^{2-}$ ion can be expressed as

$$
\rho(\mathbf{r})=\sum_{\mu, i} \sum_{\mu^{\prime}, i^{\prime}} d_{\mu i, \mu^{\prime} i^{\prime}} \chi_{\mu i} \chi_{\mu^{\prime} \iota^{\prime}}
$$

where $\left\{d_{\mu i, \mu^{\prime} i^{\prime}}\right\}$ are the elements of the symmetric density matrix and $\chi_{\mu i}$ is the $i$ th contracted real Gaussian atomic orbital centered at atom $\mu$. The terms with $\mu \neq \mu^{\prime}$ originate from overlap of atomic orbitals centered at different atoms. In the Mulliken population analysis, the contribution to the total number of electrons from these terms, i.e., $d_{\mu i, \mu^{\prime} i^{\prime}}\left\langle\chi_{\mu i} \mid \chi_{\mu^{\prime} i^{\prime}}\right\rangle$, are equally distributed to the separate atoms. Accordingly, in the present case, we separate the contributions to these terms by adding $d_{\mu i, \mu^{\prime} i^{\prime}}\left\langle\chi_{\mu i} \mid \chi_{\mu^{\prime} i^{\prime}}\right\rangle$ to the diagonal element $d_{\mu i, \mu i}$ (and $d_{\mu^{\prime} i^{\prime}, \mu i}\left\langle\chi_{\mu^{\prime} i^{\prime}} \mid \chi_{\mu i}\right\rangle$ to $\left.d_{\mu^{\prime} i^{\prime}, \mu^{\prime} i^{\prime}}\right)$. Then we have a density matrix $d_{\mu i, \mu^{\prime} i^{\prime}}^{\prime}$ block diagonalized with respect to different atoms, in terms of which the total charge density can be written approximately as

$$
\rho(\mathbf{r}) \approx \sum_{\mu} \sum_{i, i^{\prime}} d_{\mu i, \mu i^{\prime}}^{\prime} \chi_{\mu i} \chi_{\mu i^{\prime}}
$$

Consequently we write $\rho(\mathbf{r})=\Sigma_{\mu} \rho_{\mu}(\mathbf{r})$, with $\rho_{\mu}(\mathbf{r})$ $=\sum_{i, i^{\prime}} d_{\mu i, \mu i^{\prime}}^{\prime} \chi_{\mu i} \chi_{\mu i^{\prime}}$, defining the charge-density distribution of atom $\mu$. If we integrate the charge density $\rho_{\mu}(\mathbf{r})$ for each atom, the number of electrons obtained is exactly the same as given by Mulliken population analysis. The resultant ionicities for $\mathrm{Se}$ and $\mathrm{O}$ are 1.1504 and -0.7876 , respectively, very different from the +6 and -2 values for the closed-shell configurations of these atoms.

We should like to emphasize that such a separation of the total charge density of the selenate ion into components for its constituent atoms is meaningful only for the calculation of the intermolecular potentials. It is used to provide an approximate but effective representation of the interactions external to the selenate group and is not to be understood as an intramolecular assignment of electrons to the individual atoms, since these electrons are intrinsically shared by all the atoms in the group, through the Hartree-Fock calculations.

We note that, in principle, the total charge distribution of the $\mathrm{SeO}_{4}{ }^{2-}$ ion produced by the quantum-chemistry calculations depends on the configuration of this ion, as do the charge densities of the individual atoms obtained by the procedure explained above. However, because of the bond and bond-angle stiffness, the $\mathrm{SeO}_{4}{ }^{2-}$ ions do not alter their shapes drastically in the different phases or in thermal motions, even at room temperature, as will be seen from our molecular-dynamics simulations at such temperatures. Therefore we assume the ions within $\mathrm{SeO}_{4}{ }^{2-}$ to have rigid electron charge distributions and we take these distributions as those calculated for the optimized structure of $\mathrm{SeO}_{4}{ }^{2-}$, since this structure is very close to the actual structure of the selenate ions in the crystal. We should again stress that this "rigid-ion" assumption only concerns calculations of the intermolecular interactions. For the interactions within the $\mathrm{SeO}_{4}{ }^{2-}$ group, which could be much more sensitive to the charge-density variations, this assumption may be invalid. But our approach to the description of these interactions, described at the beginning of this section, automatically includes the effect of such charge-density variations.

In the Gordon-Kim model and charge distributions of the two atoms are spherical. ${ }^{13}$ The charge distributions for the atoms obtained from the separation described above do not necessarily meet this requirement, due to the unequal populations on the different components of the $p$ and/or $d$ orbitals, and therefore have to be modified further. Our method is to condense the $p$ and $d$ orbitals and to replace them by spherical orbitals that contain just the radial parts of the $p$ or $d$ orbitals. For example, for a $p$ orbital, we add together all the elements in the $3 \times 3$ block in the density matrix corresponding to the $p_{x}, p_{y}$, and $p_{z}$, orbitals to form a diagonal element for a new spherical orbital that has just the radial part of the $p$ orbital. We also add the three elements that involve one of the three components and another different orbital to make an off-diagonal element between the new spherical orbital and that particular orbital. The resultant charge distribution consists of a condensed matrix with a number of spherical orbitals, corresponding to each shell of the electronic configuration, and is therefore spherical.

Certainly, the charge densities for the individual atoms obtained from the procedure outlined above give only an approximate representation of the electron charge distribution in the molecular ion. To understand better the approximations involved, we constructed an approximate electron charge-density distribution for $\mathrm{SeO}_{4}{ }^{2-}$ by superposition of these spherical charge densities obtained for the individual atoms $\mathrm{Se}$ and $\mathrm{O}$ and compared it with the exact charge-density distribution for $\mathrm{SeO}_{4}{ }^{2-}$. We found that while the differences between the two are appreciable in regions within the $\mathrm{SeO}_{4}{ }^{2-}$, e.g., on the $\mathrm{Se}-\mathrm{O}$ bonds, 
they are very small on the outskirts of the $\mathrm{SeO}_{4}{ }^{2-}$ group. Since it is the overlap of these outskirts that contributes most to the Gordon-Kim potentials external to the $\mathrm{SeO}_{4}{ }^{2-}$ ion, our approximate charge densities for the individual atoms should be sufficiently accurate, since they are used only for the intermolecular interactions. The excellent agreements between theory and experiment to be presented later will show that this is indeed the case. While the present procedure for extracting the spherical charge densities for individual atoms appears rather crude; it is, however, direct and simple, and allows systematic improvement if necessary for other systems.

Finally, it should be noted that this method for extracting the spherical charge-density distributions for individual atoms is such that it will be exact for the charge distribution of a closed shell, as can be easily shown. This may be important, especially for atoms that contain a large number of electrons, e.g., for Se. For these atoms most of the electrons are inner core electrons that retain a closed-shell structure.

Using the resultant spherical charge-density distributions for $\mathrm{Se}$ and $\mathrm{O}$, and the closed-shell charge-density distribution for free $\mathrm{K}^{+}$, we then calculated the GordonKim pair potentials between these ions. The long-range Coulomb interactions are determined from the fractional ionicities of the Se and $\mathrm{O}$ atoms, 1.1504 for Se and -0.7876 for $\mathrm{O}$, and the ionicity +1 for $\mathrm{K}^{+}$. The shortrange potentials were calculated numerically over a range of separations from 2 to 12 a.u. for all the ion pairs; for greater separations these potentials are less than $10^{-7}$ hartree.

These numerical short-range potentials were then fitted to the analytic form employed previously ${ }^{11}$

$$
U(r)=e^{-\alpha r} \sum_{n=0}^{N} C_{n} r^{m+n}, \quad r_{c}<r<r_{t},
$$

with $r_{c}=2$ and $r_{t}=12$ a.u., to avoid numerical differentiation. It was found that polynomials of degree $N=8$ are enough to provide fits with errors of less than $0.1 \%$ at every point. To complete the fit, a core function $U(r)=A+B / r^{6}, \quad r \leq r_{c}$, and a tail function $U(r)$ $=C \exp \left(-\beta r^{2}\right), r \geq r_{t}$, were attached by requiring that the potential and its first derivative be continuous at the points of attachment.

An alternative to the present description of the intermolecular interactions is to use, rather than ion-to-ion pairwise potentials, potentials derived for the molecular groups from their unmodified charge densities as has been done for Ar-CO. ${ }^{18}$ However, this requires that the $\mathrm{SeO}_{4}{ }^{2-}$ ion be treated in the dynamics as a rigid rotor. The resultant complexities and ambiguities are such that this is clearly a worse approach: the single-atom pairwise potential is both more accurate dynamically and an unambiguous zero-order approximation that can be systematically improved if necessary.

\section{STATIC Pnam AND Pna 2 1 STRUCTURES OF $\mathrm{K}_{2} \mathrm{SeO}_{4}$}

With the intramolecular and intermolecular potentials determined, we performed static relaxation calculations for the room-temperature Pnam and low-temperature Pna $2_{1}$ structures of $\mathrm{K}_{2} \mathrm{SeO}_{4}$. These relaxations gave the crystal structures that are at extremes of the theoretical potential-energy surface-where the forces on the basis ions are zero. A comparison of the positions of the ions in these structures with the positions determined by experiment provides a sensitive test of the validity of the theoretical potential-energy surface.

First we consider the room-temperature Pnam structure of $\mathrm{K}_{2} \mathrm{SeO}_{4}$ (space group $D_{2 h}^{16}$ ). Figure 1 shows the projections of the experimental positions ${ }^{2}$ parallel to each of the three orthorhombic axes $(a=7.661 \AA$, $b=10.466 \AA$, and $c=6.003 \AA) .{ }^{2}$ The prototypic parameters for the experimental structure ${ }^{2}$ are given in Table $I$ in parentheses.

Our relaxation started from the experimental structure and was subject to the constraints of Pnam symmetry, i.e., only the parameters in the parentheses in Table I and the lengths of the lattice constants $a, b, c$ were allowed to vary. The minimization was for an infinite lattice, obtained by applying periodic boundary conditions, and followed a Newton-Raphson algorithm. The standard technique of the Ewald sum was used for the calculation of the lattice energy and forces, etc.

The relaxed structures have zero forces on the basis ions and zero forces of constraint. Projections of the ion positions in the relaxed structure are shown in Fig. 2. The lattice constants are $a=7.261 \AA, b=9.859 \AA$, and $c=5.670 \AA$. The relaxed prototypic parameters are given in Table $I$ along with the experimental values in parentheses.

As can be seen both from Fig. 2 and from the prototypic parameters given in Table I, our relaxed structure agrees very well with experiment, as far as the reduced (fractional) basis vectors are concerned. The largest difference between theory and experiment is only 0.0168 , for the $y / b$ parameter of $\mathrm{K}(2)$, which corresponds to a change of $0.1759 \AA$ in the atomic position. This discrepancy is well below the thermal fluctuation of the atomic positions at room temperature.

The lattice constants for the relaxed structure have an overall shortening of $\sim 5 \%$ from the experimental values. This has been a rather general feature for large unit-cell simulations using Gordon-Kim potentials. Since the shortenings are comparable for the three axes $(5.2 \%$ for $a, 5.8 \%$ for $b$, and $5.5 \%$ for $c$ ), they should not be important, as far as the structural phase transitions are concerned. As was pointed out in a previous study of $\mathrm{Rb}_{2} \mathrm{ZnCl}_{4},{ }^{12}$ which is isomorphous to $\mathrm{K}_{2} \mathrm{SeO}_{4}$, we believe that the incommensurate phase transitions in these isomorphs originates from a "latent" symmetry in the structure - an imperfect sixfold helical symmetry (in the Se and $K$ sublattices) caused by the inequivalence of $\mathrm{SeO}_{4}{ }^{2-}$ ions pointing along $+\mathbf{a}$ and $-\mathbf{a}$. If this were $\mathrm{ab}-$ sent and the symmetry perfect, then the projection of these atoms parallel to a axis would form a twodimensional ideal hexagonal structure with the $b / c$ ratio equal to $1.732(\sqrt{3})$, while the value of this ratio in our theoretical structure is 1.739 , in good agreement with the experimental value 1.743 . This indicates that, while the discrepancies in the absolute lengths of the axes are 


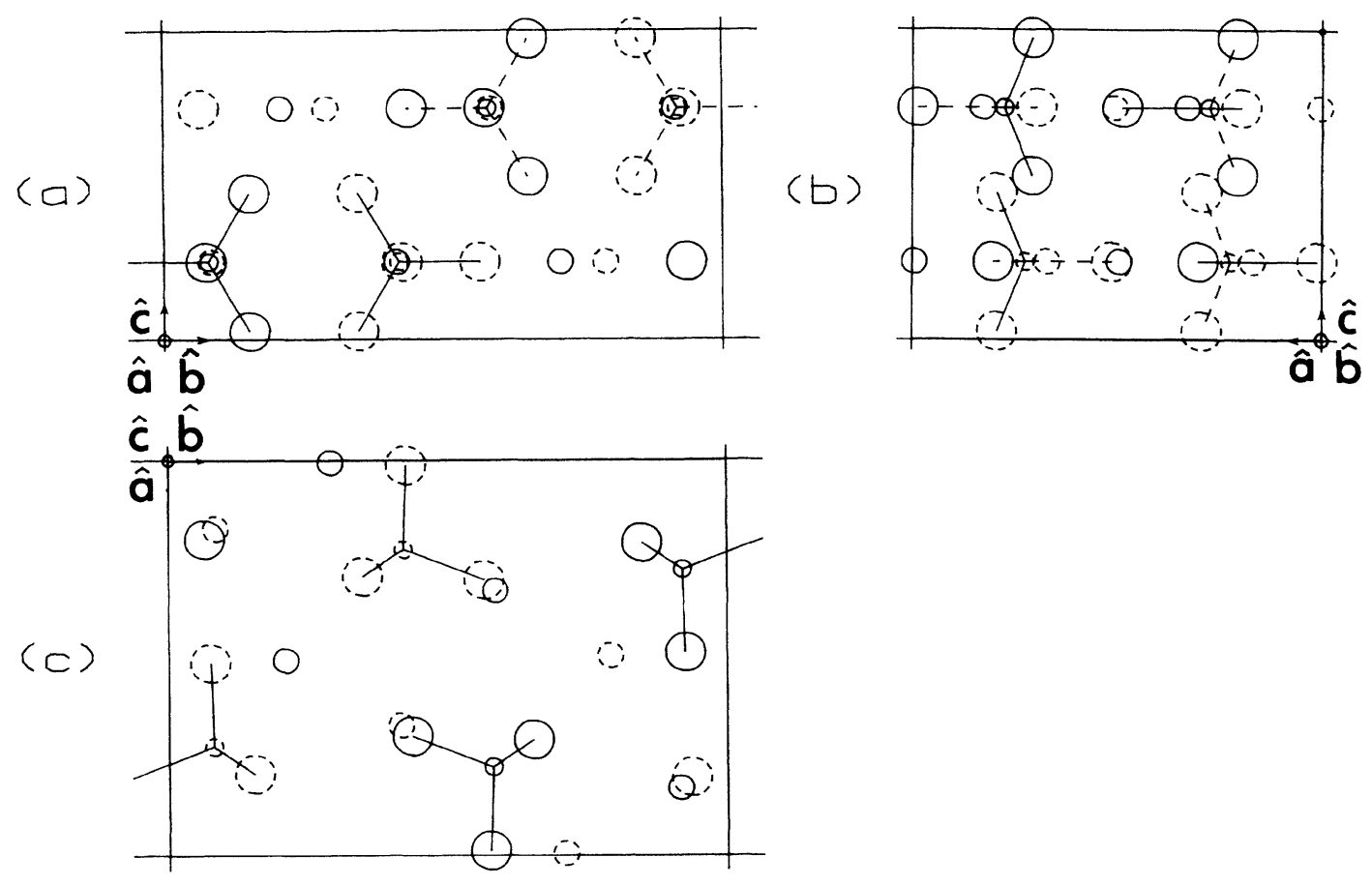

FIG. 1. Projections of the experimentally determined average structure of $\mathrm{K}_{2} \mathrm{SeO}_{4}$ in the Pnam phase. Unit vectors indicate the directions of the lattice basis vectors. A unit vector pointing out of the figure is represented by a circle and the one pointing into the figure is represented by a dot. Bonds (straight lines) connect each Se and the nearest four O ions.

significant, the near uniformly contracted theoretical structure should still preserve those basic symmetries of the system relevant to the phase transitions under study. In particular, the $\mathrm{SeO}_{4}{ }^{2-}$ ions will lie on the imperfect sixfold helices that replace their exact counterparts in the "ideal" hexagonal structure. In this situation there is no longer any reason to expect that an exact threefold repeat will render the helical component of the free energy stationary, rather than contrary, hence an incommensurate phase can develop. One can also argue this by considering the torques between adjacent molecular ions; if one examines the situation closely, it becomes apparent that, just as one has to impose vanishing surface stresses explicitly when periodic boundary conditions have been assumed; so too does one have to relax the surface torques in the emergent helices. When the helices are regular the only nonzero torques, about the helical axis, are in the surface cells and their relaxation only perturbs the sur- face structure. However, when the helices are nonuniform and distorted within a unit cell there will exist a net torque about the helical axis for each unit cell, which in the infinite lattice, has to be offset by counter torques from ions outside the helix. Clearly such a situation is metastable with respect to any structural change that will reduce these torques. This is provided at the surfaces. Clearly the "unwinding" that relaxes the surface torques for regular helices also permits a further unwinding to reduce uniformally the torques within each unit cell. This is a bulk effect and it appears as a finite uniform change in the average pitch of the helices that no longer has to be commensurate with the lattice constant.

In a previous study of $\mathrm{Rb}_{2} \mathrm{ZnCl}_{4}$ (Ref. 10) it was found that a static relaxation for the Pnam structure using Gordon-Kim potentials computed from closed-shell charge densities of ions with full ionicity gave a structure that differs from the experimental structure in several as-

TABLE I. Prototypic atomic positions for $\mathrm{K}_{2} \mathrm{SeO}_{4}$ in the relaxed Pnam structure. Experimental values (Ref. 9) are given in parentheses.

\begin{tabular}{ccccc}
\hline \hline Prototype & $x / a$ & $y / b$ & $z / c$ & $\begin{array}{c}\text { Wyckoff } \\
\text { designation }\end{array}$ \\
\hline $\mathrm{Se}$ & $0.2324(0.2242)$ & $0.4203(0.4200)$ & $\frac{1}{4}$ & $4(\mathrm{c})$ \\
$\mathrm{K}(1)$ & $0.1589(0.1705)$ & $0.0892(0.0843)$ & $\frac{1}{4}$ & $4(\mathrm{c})$ \\
$\mathrm{K}(2)$ & $0.9869(0.9943)$ & $0.6927(0.7095)$ & $\frac{1}{4}$ & $4(\mathrm{c})$ \\
$\mathrm{O}(1)$ & $0.3074(0.2931)$ & $0.3432(0.3471)$ & $0.0183(0.0271)$ & $8(\mathrm{~d})$ \\
$\mathrm{O}(2)$ & $0.3136(0.3024)$ & $0.5728(0.5644)$ & $\frac{1}{4}$ & $4(\mathrm{c})$ \\
$\mathrm{O}(3)$ & $0.0101(0.0126)$ & $0.4216(0.4251)$ & $\frac{1}{4}$ & $4(\mathrm{c})$ \\
\hline \hline
\end{tabular}




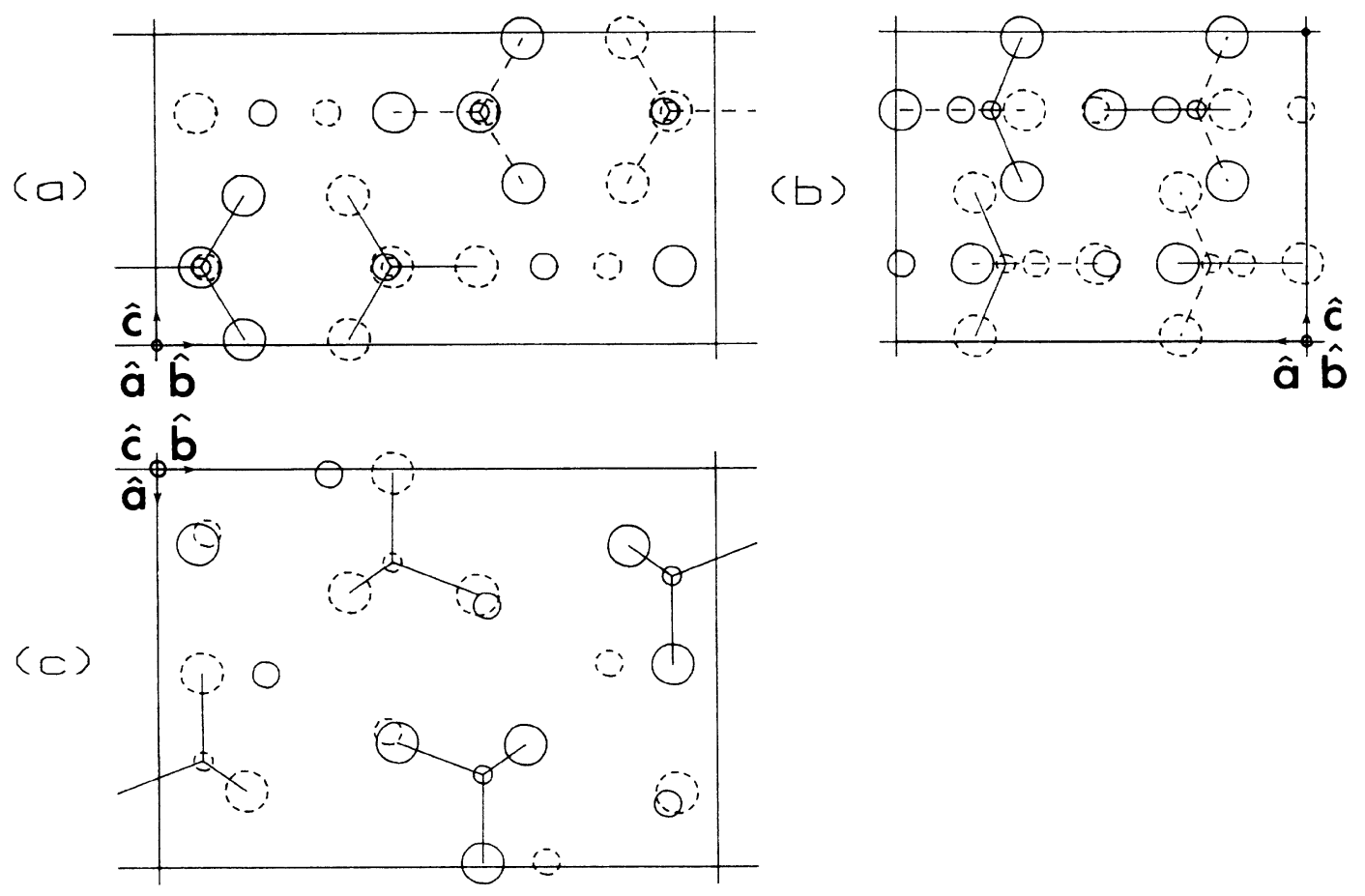

FIG. 2. Projections of the theoretical structure obtained by static relaxation with Pnam constraint.

pects: the $\mathrm{Zn}-\mathrm{Cl}$ bond lengths were $10 \%$ too large, the $\mathrm{ZnCl}_{4}{ }^{2-}$ ions were distorted from near perfect tetrahedra and rotated so that no $\mathrm{Zn}-\mathrm{Cl}$ bond was parallel to the a axis. These discrepancies also appeared for $\mathrm{K}_{2} \mathrm{SeO}_{4}$ when we used Gordon-Kim potentials computed from the closed-shell charge distributions for $\mathrm{Se}^{6+}, \mathrm{O}^{2-}$, and $\mathrm{K}^{+}$ ions. However, none of these discrepancies exists significantly in the present structure, as is evident from Fig. 2 and Table I.

Next we performed a static relaxation for the lowertemperature ferroelectric structure (space group Pna $2_{1}$ ) of $\mathrm{K}_{2} \mathrm{SeO}_{4}$ to examine further the potential-energy surface. Figure 2 shows projections of the experimental positions parallel to each of the three orthorhombic axes

TABLE II. Prototypic atomic positions for $\mathrm{K}_{2} \mathrm{SeO}_{4}$ relaxed within Pna $2_{1}$ constraints.

\begin{tabular}{ccccc}
\hline \hline & & & & \\
Prototype & $x / a$ & $/ b$ & $\begin{array}{c}\text { Wyckoff } \\
\text { designation }\end{array}$ \\
\hline $\mathrm{Se}(1)$ & $0.0772(0.0740)$ & $0.4186(0.4171)$ & $0.2436(0.2436)$ & $4(\mathrm{a})$ \\
$\mathrm{Se}(2)$ & $0.4103(0.4066)$ & $0.4182(0.4180)$ & $0.2644(0.2606)$ & $4(\mathrm{a})$ \\
$\mathrm{Se}(3)$ & $0.7441(0.7416)$ & $0.4182(0.4203)$ & $0.2438(0.2431)$ & $4(\mathrm{a})$ \\
$\mathrm{K}(1)$ & $0.0532(0.0544)$ & $0.0852(0.0800)$ & $0.2677(0.2556)$ & $4(\mathrm{a})$ \\
$\mathrm{K}(2)$ & $0.3876(0.3890)$ & $0.0888(0.0797)$ & $0.2548(0.2441)$ & $4(\mathrm{a})$ \\
$\mathrm{K}(3)$ & $0.7199(0.7225)$ & $0.0871(0.0811)$ & $0.2326(0.2296)$ & $4(\mathrm{a})$ \\
$\mathrm{K}(4)$ & $-0.0031(-0.0001)$ & $0.6943(0.7080)$ & $0.2724(0.2704)$ & $4(\mathrm{a})$ \\
$\mathrm{K}(5)$ & $0.03303(0.3327)$ & $0.6946(0.7059)$ & $0.2298(0.2344)$ & $4(\mathrm{a})$ \\
$\mathrm{K}(6)$ & $0.6640(0.6664)$ & $0.6947(0.7081)$ & $0.2462(0.2499)$ & $4(\mathrm{a})$ \\
$\mathrm{O}(1)$ & $0.1089(0.1018)$ & $0.3550(0.3486)$ & $0.0126(0.0373)$ & $4(\mathrm{a})$ \\
$\mathrm{O}(2)$ & $0.4320(0.4247)$ & $0.3526(0.3400)$ & $0.0191(0.0314)$ & $4(\mathrm{a})$ \\
$\mathrm{O}(3)$ & $0.7604(0.7545)$ & $0.3195(0.3291)$ & $0.0267(0.0278)$ & $4(\mathrm{a})$ \\
$\mathrm{O}(4)$ & $0.0918(0.0942)$ & $0.3230(0.3358)$ & $0.4663(0.4660)$ & $4(\mathrm{a})$ \\
$\mathrm{O}(5)$ & $0.4356(0.4324)$ & $0.3256(0.3393)$ & $0.4766(0.4888)$ & $4(\mathrm{a})$ \\
$\mathrm{O}(6)$ & $0.7749(0.7715)$ & $0.3588(0.3518)$ & $0.4798(0.4807)$ & $4(\mathrm{a})$ \\
$\mathrm{O}(7)$ & $0.1066(0.1012)$ & $0.5673(0.5678)$ & $0.2871(0.2608)$ & $4(\mathrm{a})$ \\
$\mathrm{O}(8)$ & $0.4400(0.4411)$ & $0.5681(0.5562)$ & $0.2870(0.2539)$ & $4(\mathrm{a})$ \\
$\mathrm{O}(9)$ & $0.7730(0.7717)$ & $0.5670(0.5618)$ & $0.1955(0.2049)$ & $4(\mathrm{a})$ \\
$\mathrm{O}(10)$ & $0.0039(0.0024)$ & $0.4287(0.4311)$ & $0.2028(0.2223)$ & $4(\mathrm{a})$ \\
$\mathrm{O}(11)$ & $0.3362(0.3386)$ & $0.4256(0.4331)$ & $0.2757(0.2952)$ & $4(\mathrm{a})$ \\
$\mathrm{O}(12)$ & $0.6706(0.6693)$ & $0.4270(0.4295)$ & $0.2789(0.2799)$ & $4(\mathrm{a})$ \\
\hline \hline
\end{tabular}


$(a=22.716 \AA, b=10.339 \AA$, and $c=5.967 \AA) .{ }^{19}$ The prototypic atomic positions for the experimental structure $^{4}$ are given in Table II in parentheses. Comparing the structure in Fig. 3 with that of Fig. 1, we see that the unit cell in the Pna $2_{1}$ structure can be considered as formed by tripling the unit cell in the Pnam structure and then slightly modulating along the a direction the orientations of the $\mathrm{SeO}_{4}{ }^{2-}$ groups and the positions of the $\mathrm{K}^{+}$ions. ${ }^{19}$ This tripling of the structure is a result of a lock-in from the incommensurate phase that exists between the Pnam and $P$ na 2 phases. ${ }^{4,19}$

With the Pna $2_{1}$ symmetry constraints we performed a static relaxation starting from the experimental structure given in Fig. 3, and obtained the relaxed structure in Fig. 4 with the prototypic parameters given in Table II. The lengths of the lattice axes in the relaxed structure are $a=21.752 \AA, b=9.845 \AA$, and $c=5.715 \AA$, again shorter than the experimental values $(4.2 \%$ for $a, 4.8 \%$ for $b$, and $4.2 \%$ for $c$ ) but no worse so than for the Pnam phase.

As can be seen from comparing the structure in Fig. 4 with that in Fig. 3, our relaxation gives a close fit to the experimental data. The largest discrepancy in the reduced basis positions is (cf. Table II) 0.033 for the $z$ coordinate of $\mathrm{O}(8)$, which would mean an actual displacement
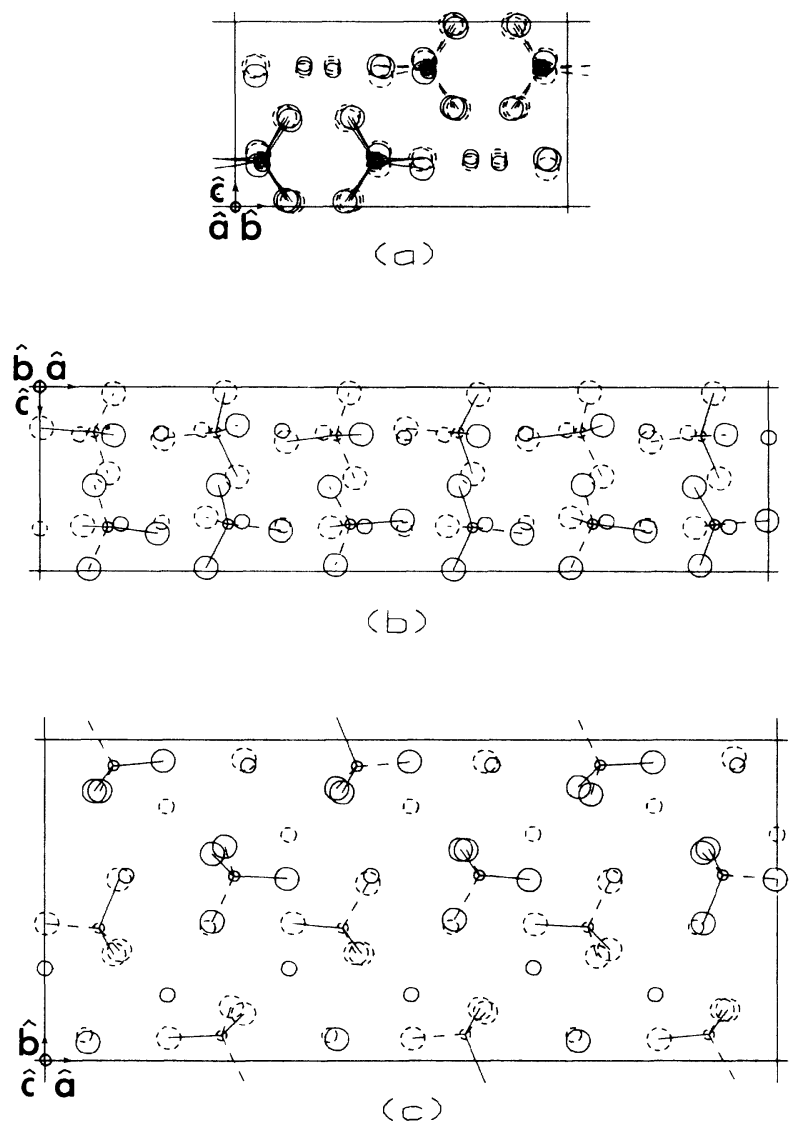

FIG. 3. Projections of the experimentally determined average structure of $\mathrm{K}_{2} \mathrm{SeO}_{4}$ in the $P n a 2_{1}$ phase. Note that the a axis is approximately triple that in the Pnam structure in Fig. 1.
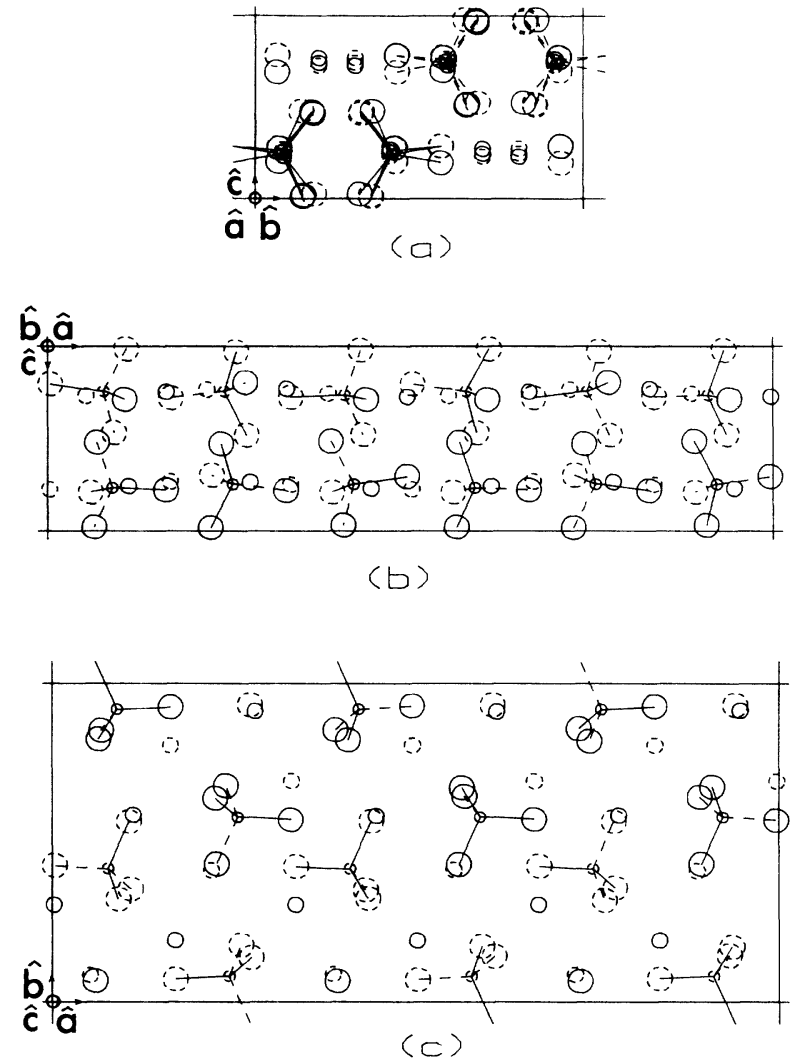

FIG. 4. Projections of the theoretical structure obtained by static relaxation with $P n a 2_{1}$ constraint.

of $0.2 \AA$ if the experimental lattice constants are used. This is well under the half width of the thermal fluctuation $\left(0.34 \AA\right.$ ) found by experiment. ${ }^{19}$ Overall the basic modulation in the experimental structure is closely reproduced, although the amplitude of the modulation is slightly larger, as is to be expected from a comparison of the static modulation with the experimental dynamical average.

The theoretical Pna $2_{1}$ structure of $\mathrm{K}_{2} \mathrm{SeO}_{4}$ in Fig. 4 possesses a spontaneous polarization along the $\mathrm{c}$ direction as found by experiment. ${ }^{20}$ Its magnitude is only $0.023 \mu \mathrm{C} / \mathrm{cm}^{2}$, smaller than the experimental value of $0.07 \mu \mathrm{C} / \mathrm{cm}^{2}$ at $80 \mathrm{~K}$. Again such a direct comparison of the static and average values must be made with caution, since the size and sense of the electronic contribution to the spontaneous polarization could be important.

\section{DYNAMICS SIMULATIONS OF THE PHASE TRANSITION}

Having successfully reproduced the static Pnam and Pna 2 1 structures of $\mathrm{K}_{2} \mathrm{SeO}_{4}$, we then performed molecular-dynamics calculations to simulate directly the phase transition from the former structure to the latter. By following the motions of the ions in real time, such simulations describe realistically the structural changes in the system as the temperature is varied and therefore provide a further test of the theoretical potential-energy surfaces, especially of the topology of these surfaces that 
governs the phase transition.

The sample for our simulation consisted of twelve of the Pnam unit cells of $\mathrm{K}_{2} \mathrm{SeO}_{4}$, in total 336 ions. This supercell was obtained by first tripling the unit cell in the a direction to obtain a cell that is consistent with the unit cell of the Pna $2{ }_{1}$ ferroelectric structure. Such tripling is obviously necessary to allow the phase transition to occur. Then the tripled cell was doubled both in $\mathbf{b}$ and $\mathbf{c}$ directions to lessen artificial correlations in those two directions. Our simulations follow a constant- (zero-) pressure algorithm ${ }^{21}$ and are made for infinite samples by employing periodic boundary conditions. To make the simulations as realistic as possible, no restriction was applied (besides the periodic boundary condition) to the supercell, i.e., all of the ion positions and all of the lattice vectors were allowed to change during the simulations. The time increment between two successive steps in the simulations was $0.005 \mathrm{ps}$, which was found to be sufficiently short.

Starting from the experimental Pnam structure, the sample was first sufficiently relaxed to be as free of experimental information as possible, while it was maintained at $T=300 \mathrm{~K}$. We then performed a molecular-dynamics "quench" by gradually reducing the total kinetic energy of the sample in stages to zero. At each step the sample was allowed to equilibrate for 2 ps and the average temperature and average positions of the ions for this temperature were calculated. We found that we could indeed reproduce realistically the experimentally observed Pnam-Pna $2_{1}$ phase transition.

Figures 5 and 6 shows the structures at $T=300$ and $57 \mathrm{~K}$, respectively, both averaged over 2 ps. In these figures, the average $\mathrm{Se}-\mathrm{O}$ bonds are indicated by straight lines and the thermal motions of the ions are given by ellipsoids centered at the average positions.

Clearly the structure in Fig. 5 is virtually the same as the experimental Pnam structure. The supercell is orthorhombic with the average lattice constants $a=21.917 \AA$, $b=19.859 \AA$, and $c=11.488 \AA$. In particular, the averaged structure clearly maintains the threefold repeat along $\mathbf{a}$ and twofold repeat along $\mathbf{b}$ and $\mathbf{c}$, within the precision of the simulation. If the supercell is divided to 12 cells, each of them fits very closely the experimental Pnam structure in Fig. 1, with the lattice axes, as expected, shorter than the experimental values by $4.6 \%$ for $a$, $4.9 \%$ for $b$, and $4.3 \%$ for $c$. (Note these fits are slightly better than those obtained in Sec. III from static relaxation.)

The large (about \pm 0.5 a.u.) amplitudes of the thermal motions of some of the oxygen ions, as seen in Fig. 5, indicate that the crystal at this temperature is actually in a dynamical disordered state. The shapes of thermal ellipsoids show that this disorder is associated with the rotations of the selenate groups. In a previous Raman measurement of the internal mode frequencies, ${ }^{22}$ more lines were found than predicted by the conventional factor group analysis for the perfect Pnam symmetry. A hypothesis was then made that the selenate ions were orientationally disordered; this is now clearly confirmed by our present simulation.

When the temperature was lowered to $57 \mathrm{~K}$, the sys- tem transformed to the structure shown in Fig. 6. Comparing it with the room temperature structure in Fig. 5, we see that while double periodicity along $\mathbf{b}$ and $\mathbf{c}$ directions was preserved, modulation occurred along the a direction, resulting in the loss of the original threefold periodicity. Evidently the resultant tripled structure closely fits the $P n a 2_{1}$ structure of $\mathrm{K}_{2} \mathrm{SeO}_{4}$. The basic modulation again agrees well with that in Fig. 3, although the amplitudes are again slightly larger. In fact, when further cooled to $T=0 \mathrm{~K}$, this structure agrees closely with the theoretical Pna $2_{1}$ structure in Fig. 4 both in ionic positions and potential energy.

Having shown that our molecular-dynamics (MD) simulations can indeed reproduce the transition of $\mathrm{K}_{2} \mathrm{SeO}_{4}$ from its Pnam phase to the Pna $2_{1}$ phase, we now
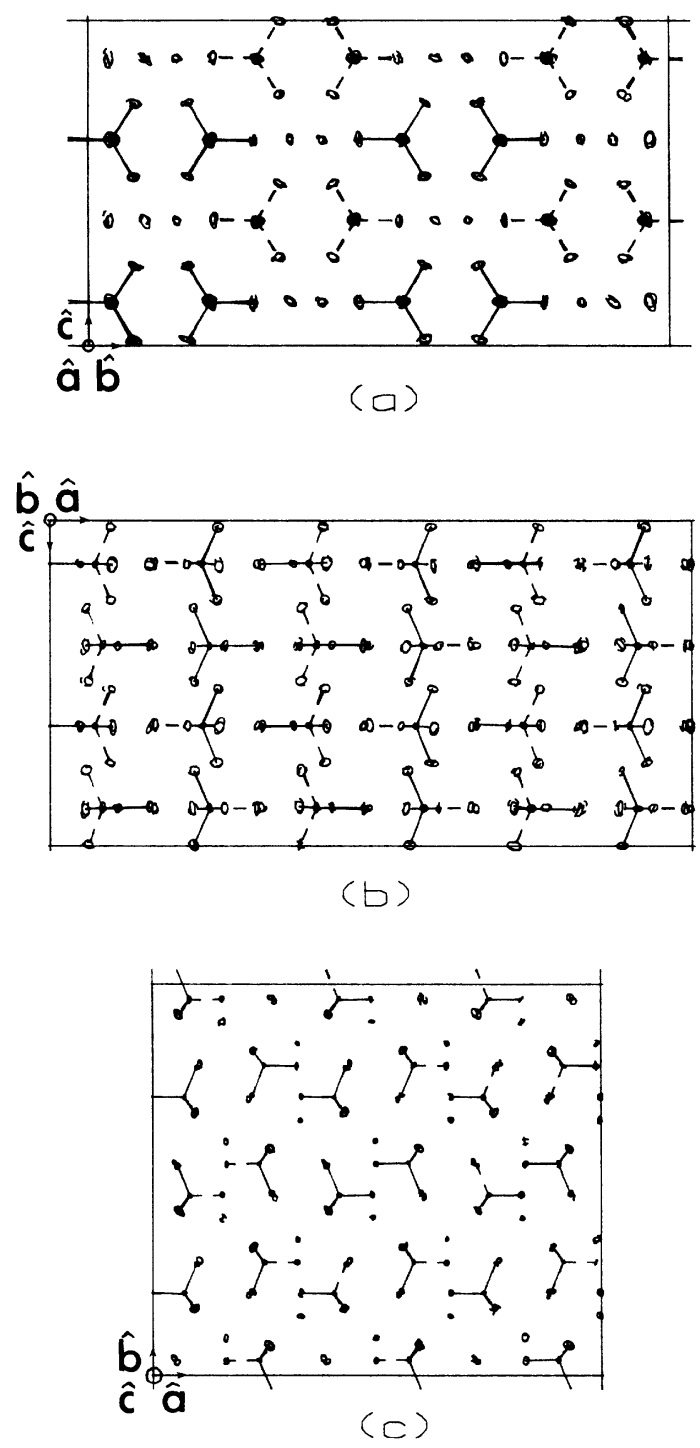

FIG. 5. Projections of the atomic positions in the average structure at $T=300 \mathrm{~K}$ obtained in the $3 \mathbf{a} \times 2 \mathbf{b} \times 2 \mathbf{c}$ supercell molecular-dynamics simulation. The ellipsoids centered at the atomic positions indicate the amplitude of the thermal motions of the ions. 
seek to monitor the transition more precisely. Ideally, following the order parameter of a transition as a function of temperature is the best way to locate the transition temperature. However, it was shown experimental$1 y^{4}$ that the primary order parameter is the amplitude of the lattice distortion that is characterized by a wave vector $\mathbf{q}=\frac{1}{3}(1-\delta) \mathbf{a}^{*}$ in the Pnam phase, with $\mathbf{a}^{*}$ being the first reciprocal-lattice vector along the [100] direction. Even if $\delta=0$, this order parameter would be difficult to calculate for a supercell that contains many Pnam cells and a large number of ions, and therefore cannot be used. However, since the modulation that induces the phase transition involves the rotations of the $\mathrm{SeO}_{4}{ }^{2-}$ ions (cf. Figs. 5 and 6), the deviations of the $\mathrm{Se}-\mathrm{O}$ bonds from their orientations in the Pnam structure can be employed
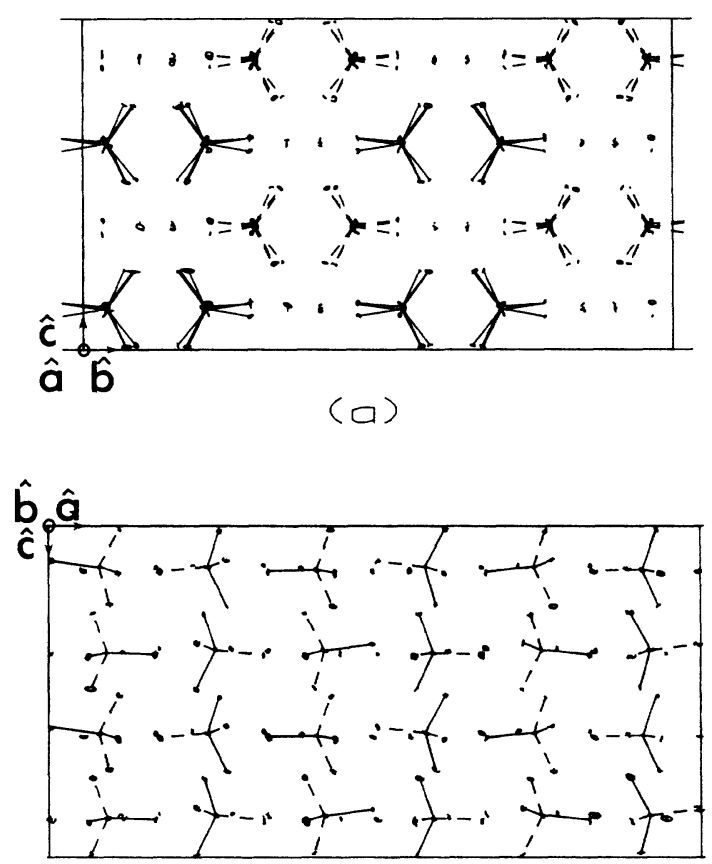

(b)

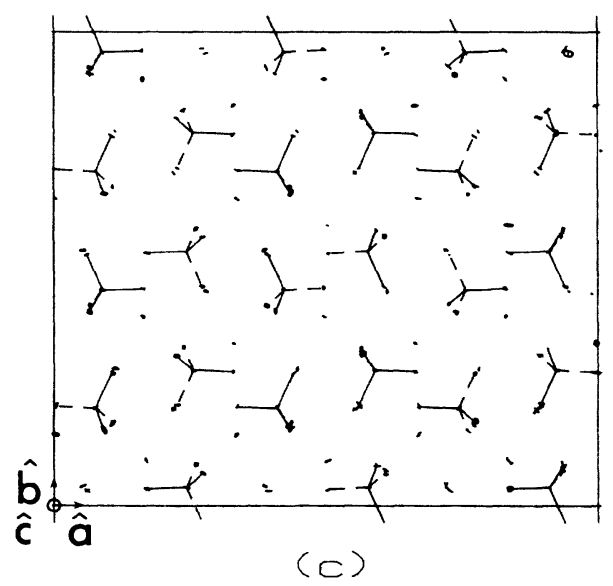

FIG. 6. Structure of $\mathrm{K}_{2} \mathrm{SeO}_{4}$ at $T=57 \mathrm{~K}$ obtained from the supercell molecular-dynamics "quenching."

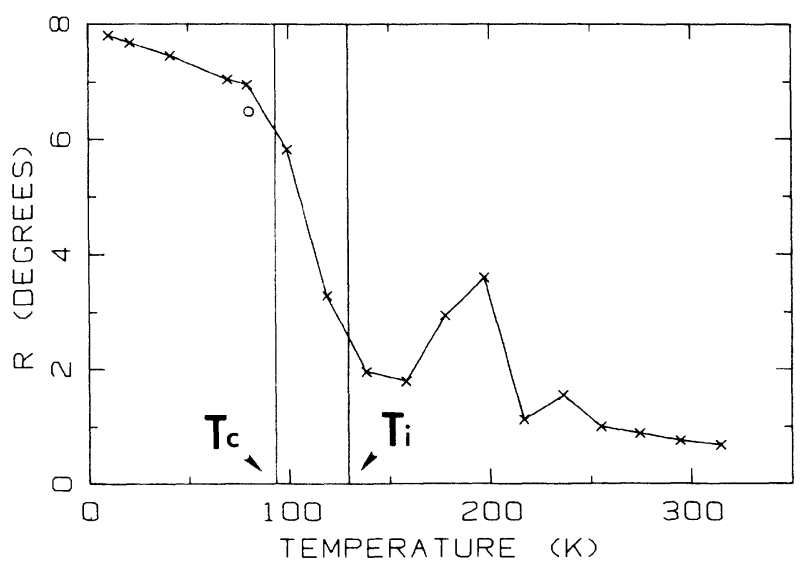

FIG. 7. Theoretical $(X)$ and experimental $(O)$ order parameter values as function of temperature.

to monitor the phase transition. Thus we calculated the rms values of this deviation by comparing the average structures at different temperatures with the static Pnam structure and employed that as an order parameter. To obtain better statistics, we again increased our supercell size to $3 a \times 2 b \times 4 c$ ( 672 ions), and extended the averaging time from 2 to $40 \mathrm{ps}$. The resultant order parameter $R$ is plotted in Fig. 7 as a function of temperature, along with the value of $R$ for the experimental Pna $2_{1}$ structure at 80 K. ${ }^{19}$

Despite the unavoidable noise, even at room temperature, Fig. 7 clearly shows that the onset of the transition lies in the range $120-140 \mathrm{~K}$, which corresponds closely to that of the incommensurate phase transition, $\left(T_{i}=129.5 \mathrm{~K}\right)$, and the transition is virtually complete at about the lock-in ferroelectric transition temperature $T_{c}=93 \mathrm{~K}$, where our order parameter is in close agreement with the experimental value. The behavior of $R$ at about $200 \mathrm{~K}$ was observed in several simulations; it is probably a dynamical, but slow, precursor to the main transition. To clarify this point completely would require an even longer computation for a still larger number of ions, and this is beyond our computing resources and would in any case, not be warranted by the minor significance of this point.

\section{DISCUSSION}

The excellent agreement between theory and experiment described in Secs. III and IV leads us to conclude that we have indeed found a very realistic $a b$ initio potential-energy surface for $\mathrm{K}_{2} \mathrm{SeO}_{4}$. We shall now discuss how this potential-energy surface provides a firstprinciples explanation of the driving mechanism of the phase transitions in $\mathrm{K}_{2} \mathrm{SeO}_{4}$.

We first calculated the dispersion curves for the theoretical relaxed Pnam structure shown in Fig. 2 in order to examine the possible instabilities allowed by the potential-energy surface for this configuration of the system. In a previous experimental study ${ }^{4}$ of $\mathrm{K}_{2} \mathrm{SeO}_{4}$ by neutron scattering, temperature-dependent dispersion 
curves were measured for $\mathrm{K}_{2} \mathrm{SeO}_{4}$ in its Pnam phase near and above $T_{i}$. It was found that the lowest-lying $\Sigma_{2}$ transverse optic branch propagating along the [100] direction softens as temperature is lowered. The minimum in this branch was at $q=0.31 \mathrm{a}^{*}$ and approached zero at the incommensurate transition temperature $T_{i}$, thus revealing the soft-transverse-optic mode responsible for driving the phase transition. This mechanism was studied by lattice-dynamical calculations within the quasiharmonic approximation ${ }^{5}$ using potentials determined from the static equilibrium constraints and the experimental zone-center frequencies. This study successfully reproduced the softening of the $\Sigma_{2}$ optical phonon with the lowest soft mode in the vicinity of $\mathbf{q}=\mathbf{a}^{*} / 3$. Thus it is of interest to see what dispersion curves are produced by our present $a b$ initio potentials. Although our calculation was made for the static structure and therefore cannot be directly compared with the experimental data, ${ }^{4}$ it does, however, reveal the nature of the potential surfaces and possible competing instabilities.

This lattice-dynamics calculation was performed for the [100] $(\Sigma)$ direction and the lowest eight branches of the dispersions are shown in Fig. 8 (the higher branches are basically irrelevant to the discussion). This figure displays the angular frequency squared $\left(\omega^{2}\right)$ divided by $\omega$ versus wave vector q; negative values therefore represent

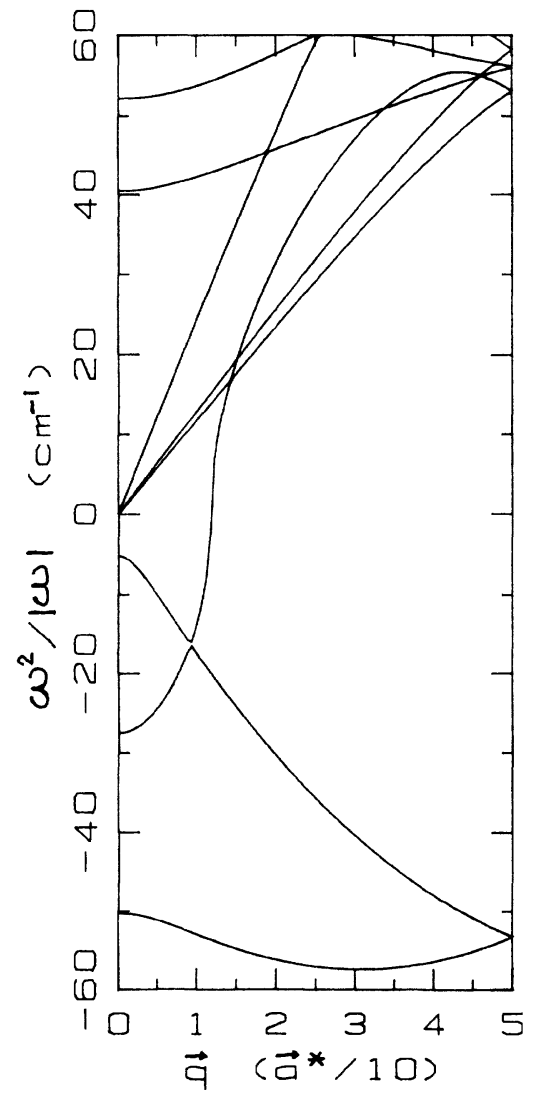

FIG. 8. Dispersion curves for the theoretical Pnam structure in Fig. 2. imaginary frequencies.

The appearance of several negative branches in Fig. 8 indicates that the theoretical Pnam structure is actually unstable. This seems surprising since the Pnam structure was reached by our static relaxation, and all the forces on the ions and the stresses were zero for this structure. The probable explanation is that the Pnam structure is not at an absolute minimum but is at a fairly broad saddle point on the potential-energy surface.

The lowest branch in Fig. 8 has $\Sigma_{2}$ symmetry and does meet a $\Sigma_{3}$ branch at the zone boundary. This at least matches partially with experiment, ${ }^{4}$ although the $\Sigma_{3}$ branch here is not an acoustic phonon and is also negative over the whole range. As pointed out previously, the present calculation does not include the temperature effects, and it is misleading to compare the present results either with experiment ${ }^{4}$ or with the previous theoretical study, ${ }^{5}$ where temperature effects were included by the quasiharmonic approximation. However, Fig. 8 does show that the absolute minimum, representing the dominant instability, occurs at $\mathbf{q}=0.304 \mathbf{a}^{*}$, very close to the experimental soft phonon wave vector $q=0.31 a^{*}$. Thus as far as the symmetry of the instability is concerned, our calculation agrees well with experiment. This result is complementary to our earlier supercell MD simulation of the cell-tripling transition, in the sense that it demonstrates, in some measure, that the tripling is not a mere consequence of the choice of the supercell.

To see if the Pnam structure has similar instabilities along other directions, we calculated the dispersion curves along both the $\mathbf{b}$ and $\mathbf{c}$ directions. We found that the lowest unstable points are at the zone center. Since this is obviously a direct consequence of the negative values at the zone center in Fig. 8, there are apparently no new instabilities. We also calculated the dispersion relations for the Pna $2_{1}$ structure and found no imaginary branches. This is to be expected since this structure is stable down to zero temperature against moleculardynamics relaxations.

The lowest $\Sigma_{2}$ branch in the dispersion shown in Fig. 8 has negative values over the whole range, indicating multiple instabilities in the system, rather than at a single unstable point near $q=\frac{1}{3} \mathbf{a}^{*}$, as found by experiment. The most interesting of these is at the zone center, involving motions of the ions without changing the periodicity of the lattice. Since the normal coordinate analysis shows that the $\Sigma_{2}$ branch involves essentially rotations of the selenate groups, it is instructive to study the zone-center instability to characterize these rotations which are, after all, the basic origin of the instability in the system.

We thus performed molecular-dynamics relaxation for a single cell of $\mathrm{K}_{2} \mathrm{SeO}_{4}$, without any constraints besides the periodic boundary condition, which only permits the zone-center instabilities. When we cooled the sample to $T=0 \mathrm{~K}$, a new stable structure, distinct from the Pnam structure, in Fig. 1, appeared which has a monoclinic lattice with the selenate groups rotated coherently. Of particular interest is that when we repeated this relaxation several times, we found that we did not always reproduce the same structure, but reached another energetically exactly equivalent structure that is also monoclinic and is a 
mirror image of the other structure. In Fig. 9 we show the projections of ions along the a directions in these two structures. If we compare Fig. 9 with Fig. 1(a), the projection of ions along the a direction in the Pnam structure, we see that the two new structures in Fig. 9 are formed by rotations of the selenate ions and shifts of the potassium ions from the Pnam positions. While the shift amplitudes are equal in both, the signs of the displacements are opposed. The potential energies of both these structures are exactly equal at $12.3 \mathrm{meV}$ per formula unit below that of the theoretical Pnam structure.

Thus we conclude that for a single Pnam cell the potential-energy surface has a double-well shape with the Pnam structure at the top and the new structures in Fig. 9 , respectively, at the bottoms of the two wells. When the periodicity along the a direction is changed, this double well generates a multiple-well structure on the total potential-energy surface, which is the origin of the Pnam-Pna $2_{1}$, cell-tripling transition. (We also checked by MD that the single-cell double-well structure was not affected by periodicity changes along the $\mathbf{b}$ and $\mathbf{c}$ directions.)

To illustrate better this double-well potential-energy surface, we then calculated the lattice potential energies as we "stepped" from the Pnam structure to one of the two new structures in Fig. 9, say [Fig. 9(a)], by changing each coordinate by $2.5 \%$ during each step. In Fig. 10 we show these energies as a function of the fractional changes in coordinate $d$. When we performed the similar calculations for stepping from the Pnam structure to the
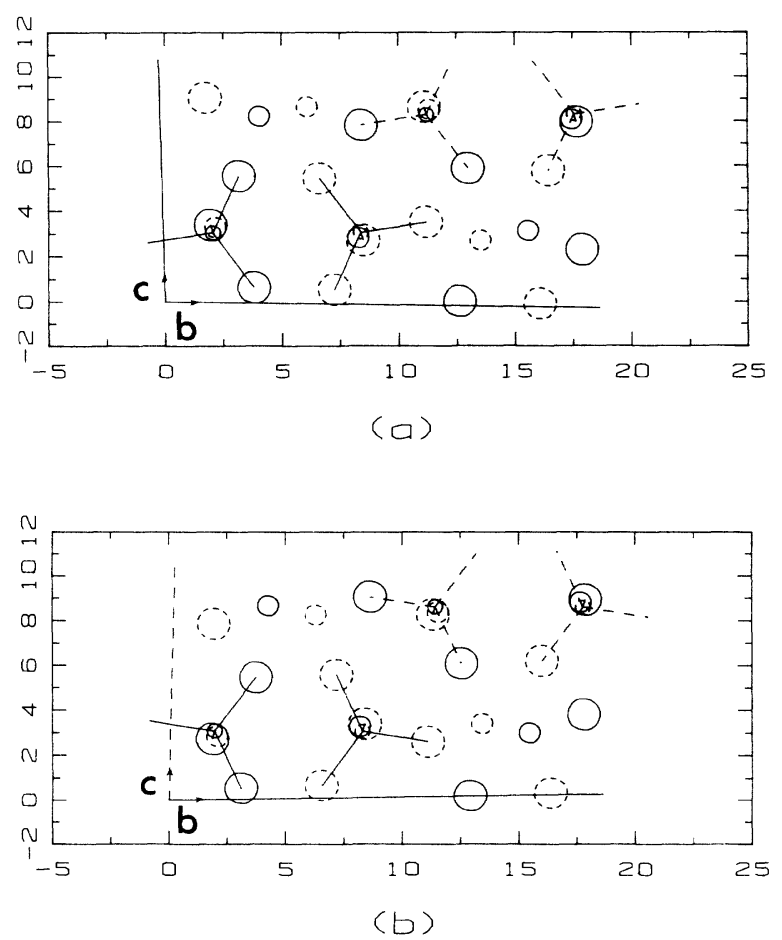

FIG. 9. bc cross sections of the two energetically equivalent singlet structures of $\mathrm{K}_{2} \mathrm{SeO}_{4}$ obtained from single-cell molecular-dynamics relaxations. The lattice axes are given in atomic units.

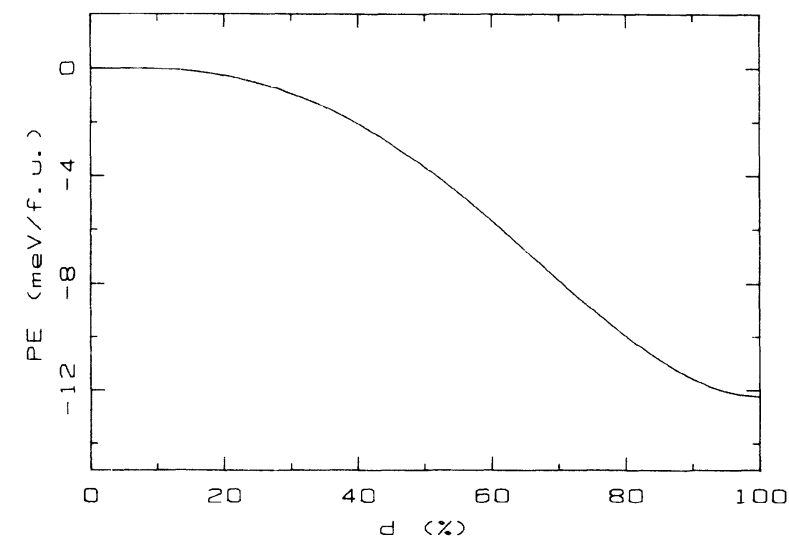

FIG. 10. Lattice potential energy (meV per formula unit) as a function of the fractional displacement from the Pnam structure $(d=0 \%)$ to the singlet structure in Fig. $9(\mathrm{a})(d=100 \%)$.

other new structure [Fig. 9(b)], i.e., using negative values for fractional changes $d$, we obtained identical results to those in Fig. 10. This conclusively demonstrates that we indeed have a double-well type of energy surface.

We then performed similar calculations of the potential energy as we "stepped" from the Pnam structure to the Pna 21 structure, and show these energies in Fig. 11. Since the Pna 2, structure can be formed from the Pnam structure in three different ways corresponding to the three equivalent ways of defining the unit cell in the Pna 2 1 structure, the potential surface of Fig. 11 is triply degenerate. While Figs. 10 and 11 display the basic features of the potential-energy surfaces, they should not be taken too literally, since the linear and equal stepping between the structures may not be the best path for the real system. For example, the very shallow dips around $d=0$ in Fig. 11 could be misleading and should therefore be ignored. It can be seen, by comparing Fig. 10 with Fig. 11, that the new singlet structures in Fig. 9 have a minimum potential energy, lower than the Pna $2_{1}$ struc-

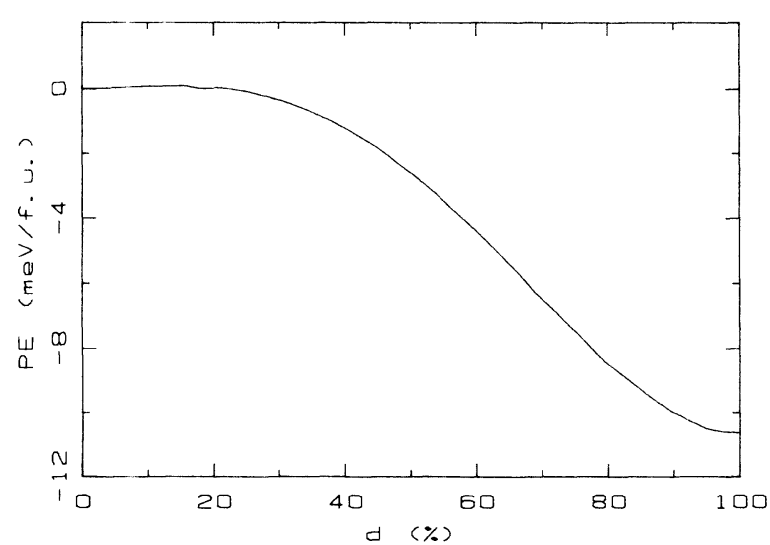

FIG. 11. Lattice potential energy (meV per formula unit) as a function of the fractional displacement from the Pnam structure $(d=0 \%)$ to the tripled $P n a 2_{1}$ structure $(d=100 \%)$. 
ture by about $1.6 \mathrm{meV}$ per formula unit. However, our supercell molecular-dynamics "quenching" did not find either of these singlet structures, but rather selected the tripled Pna $2_{1}$ structure. This is not surprising if we more closely examine the quenching process. When the temperature is above $\sim 130 \mathrm{~K}$, the small $(1.6-\mathrm{meV})$ difference in static energy between the two structures is negligible: thus the singlet and the triplet structure are effectively degenerate. Consequently as $k T$ becomes less than $\sim 10-15 \mathrm{meV}$, and the Pnam structure begins to transform, the state selected will be that of higher entropy. Clearly, this is the Pna 2, phase in which, for example, the $\mathrm{SeO}_{4}{ }^{2-}$ ions have three rather than one possible orientations. Subsequently, for the Pna $2_{1}$ structure to transform to the rotated selenate single-cell phase, it requires that at least one of the tetrahedra switch through the Pnam configuration and as the system is cooled below $k T \sim 10 \mathrm{meV}$, this becomes increasingly difficult. When $k T \sim 1-2 \mathrm{meV}$ (when the single phase would become preferred energetically), the transformation is frustrated by the $\sim 10-\mathrm{meV}$ activation barrier and the Pna2 1 phase persists.

\section{CONCLUSION}

In summary, we have developed a new approach to first-principles simulations specifically for ionic molecular solids, and using this approach we studied the phase transitions in $\mathrm{K}_{2} \mathrm{SeO}_{4}$. By starting from $a b$ initio quantumchemistry calculations for the whole molecular ion we obtained an electron charge-density distribution that includes self-consistently possible electron covalency within the molecular ion. From these distributions, $a b$ initio interionic potentials were obtained by Taylor expansion of the energy for the intramolecular interactions, and by the Gordon-Kim electron-gas model for the intermolecular interactions. Using these potentials we successfully simulated both the Pnam and the Pna $2_{1}$ structures, and the phase transition between them, obtaining a transition temperature in good agreement with experiment. We also performed first-principles lattice-dynamics studies of $\mathrm{K}_{2} \mathrm{SeO}_{4}$ that revealed that the most instable point of the Pnam structure is in the neighborhood of $\mathbf{q}=\frac{1}{3} \mathbf{a}^{*}$, very close to that observed experimentally. By investigating the potential-energy surfaces pertinent to the phase transition, we found that a rotational double-well type of motion is the basic origin of the instability in the system.

\section{ACKNOWLEDGMENTS}

This work was supported by the U.S. Army Research Office (Research Triangle Park, NC). We should also like to acknowledge many helpful discussions with Dr. P. J. Edwardson and Dr. D. P. Billesbach.
${ }^{1}$ H. Z. Cummins, Phys. Rep. 185, 211 (1990).

${ }^{2}$ A. Kálmán, J. S. Stephens, and D. W. J. Cruickshank, Acta Crystallogr. Sec. B 26, 1451 (1970).

${ }^{3}$ N. Ohama, Mater. Res. Bull. 9, 283 (1974).

${ }^{4}$ M. Iizumi, J. D. Axe, G. Shirane, and K. Shimoaka, Phys. Rev. B 15, 4392 (1977).

${ }^{5}$ M. S. Haque and J. R. Hardy, Phys. Rev. B 21, 245 (1980).

${ }^{6}$ L. L. Boyer, Phys. Rev. Lett. 42, 584 (1979). A fuller account is given in Phys. Rev. B 23, 3673 (1981).

${ }^{7}$ L. L. Boyer, Phys. Rev. Lett. 45, 1858 (1980); 46, 1172 (1981).

${ }^{8}$ L. L.Boyer and J. R. Hardy, Phys. Rev. B 24, 2527 (1981).

${ }^{9}$ L. L. Boyer, J. Phys. C 17, 1825 (1984).

${ }^{10}$ V. Katkanant, P. J. Edwardson, J. R. Hardy, and L. L. Boyer, Phys. Rev. Lett. 57, 2033 (1986).

${ }^{11}$ P. J. Edwardson, V. Katkanant, J. R. Hardy, and L. L. Boyer, Phys. Rev. B 35, 8479 (1987).

${ }^{12}$ V. Katkanant, P. J. Edwardson, and J. R. Hardy, Phase Tran- sitions 15, 103 (1989).

${ }^{13}$ R. G. Gordon and Y. S. Kim, J. Chem. Phys. 56, 3122 (1971).

${ }^{14}$ H. M. Lu and J. R. Hardy, Phys. Rev. Lett. 64, 661 (1990).

${ }^{15}$ M. J. Frisch et al., GAUSSIAN86 (Carnegie-Mellon Quantum Chemistry Publishing Unit, Pittsburgh, PA, 1984).

${ }^{16}$ E. Clementi and C. Roetti, At. Data Nucl. Data Tables 14, 177 (1974).

${ }^{17}$ R. S. Mulliken, J. Chem. Phys. 23, 1833 (1955).

${ }^{18}$ G. A. Parker and R. T. Pack, J. Chem. Phys. 69, 3268 (1978).

${ }^{19}$ N. Yamada, Y. Ono, and T. Ikeda, J. Phys. Soc. Jpn. 53, 2565 (1984).

${ }^{20}$ K. Aiki, K. Fukuda, H. Koga, and T. Kobayashi, J. Phys. Soc. Jpn. 28, 389 (1970).

${ }^{21}$ M. Parrinello and A. Rahman, Phys. Rev. Lett. 45, 1196 (1980).

${ }^{22}$ V. Katkanant, J. R. Hardy, and F. G. Ullman, Ferroelectrics 82, 185 (1988). 